\title{
Advanced Hodgkin lymphoma in the East of England: a 10-year comparative analysis of outcomes for real-world patients treated with ABVD or escalated-BEACOPP, aged less than 60 years, compared with 5-year extended follow-up from the RATHL trial
}

\author{
James Russell ${ }^{1}$ (D) Angela Collins ${ }^{2} \cdot$ Alexis Fowler $^{3} \cdot$ Mamatha Karanth $^{4} \cdot$ Chandan Saha $^{1} \cdot$ Suzanne Docherty ${ }^{2}$. \\ Joseph Padayatty ${ }^{1} \cdot$ Kyaw Maw ${ }^{5}$. Isabel Lentell ${ }^{4} \cdot$ Lisa Cooke $^{6}$. Andrew Hodson ${ }^{7}$. Nimish Shah ${ }^{2} \cdot$ Shalal Sadullah $^{5}$. \\ Nicholas Grigoropoulos ${ }^{1}$. Wendi Qian ${ }^{1} \cdot$ Amy A. Kirkwood ${ }^{8}$ - Benjamin J. Uttenthal ${ }^{1} \cdot$ Peter Johnson $^{9}$. \\ George A. Follows ${ }^{1}$ (D)
}

Received: 10 October 2020 / Accepted: 10 February 2021 / Published online: 27 February 2021

(C) The Author(s) 2021

\begin{abstract}
Treatment with ABVD (doxorubicin, bleomycin, vinblastine, and dacarbazine) or escalated(e)-BEACOPP (bleomycin, etoposide, doxorubicin, cyclophosphamide, vincristine, procarbazine, and prednisolone) remains the international standard of care for advanced-stage classical Hodgkin lymphoma (HL). We performed a retrospective, multicentre analysis of 221 non-trial ("real-world") patients, aged 16-59 years, diagnosed with advanced-stage HL in the Anglia Cancer Network between 2004 and 2014, treated with ABVD or eBEACOPP, and compared outcomes with 1088 patients in the Response-Adjusted Therapy for Advanced Hodgkin Lymphoma (RATHL) trial, aged 18-59 years, with median follow-up of 87.0 and 69.5 months, respectively. Real-world ABVD patients ( $n=177$ ) had highly similar 5-year progression-free survival (PFS) and overall survival (OS) compared with RATHL (PFS 79.2\% vs $81.4 \%$; OS $92.9 \%$ vs 95.2\%), despite interim positron-emission tomography-computed tomography (PET/CT)-guided dose-escalation being predominantly restricted to trial patients. Real-world eBEACOPP patients $(n=44)$ had superior PFS (95.5\%) compared with real-world ABVD (HR 0.20, $p=0.027$ ) and RATHL (HR 0.21, $p=0.015$ ), and
\end{abstract}

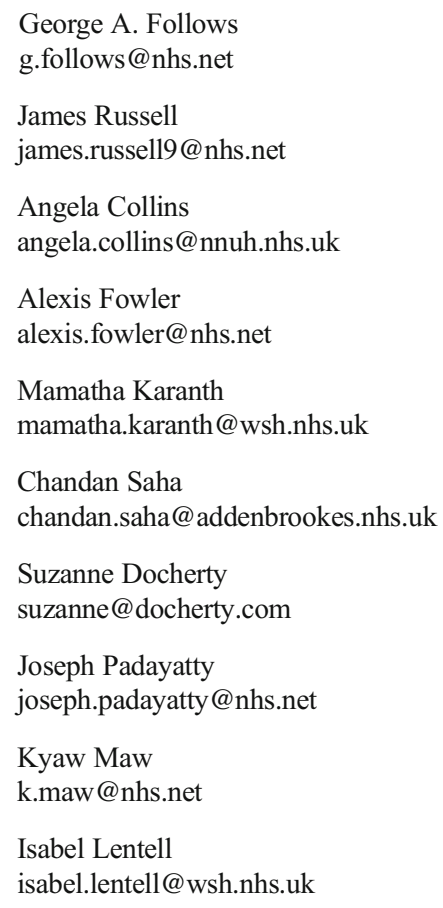

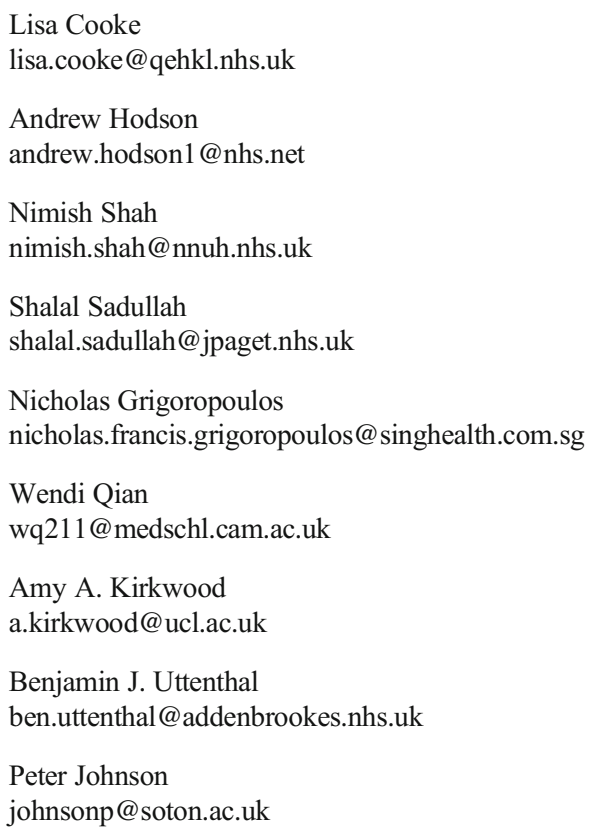

Extended author information available on the last page of the article 
superior OS for higher-risk (international prognostic score $\geq 3$ [IPS 3+]) patients compared with real-world IPS 3+ ABVD (100\% vs $84.5 \%, p=0.045$ ), but not IPS $3+$ RATHL patients. Our data support a PFS, but not OS, advantage for patients with advancedstage HL treated with eBEACOPP compared with ABVD and suggest higher-risk patients may benefit disproportionately from more intensive therapy. However, increased access to effective salvage therapies might minimise any OS benefit from reduced relapse rates after frontline therapy.

Keywords Hodgkin lymphoma $\cdot$ Advanced stage $\cdot$ Response-adapted therapy $\cdot$ Real-world data $\cdot$ ABVD $\cdot$ Escalated BEACOPP

\section{Introduction}

Treatment with ABVD (doxorubicin, bleomycin, vinblastine, and dacarbazine) or escalated(e)-BEACOPP (bleomycin, etoposide, doxorubicin, cyclophosphamide, vincristine, procarbazine, and prednisolone) remains the international standard of care for the management of adult patients with advanced-stage classical Hodgkin lymphoma (HL). In 2009, 10-year follow-up data from the German Hodgkin Study Group (GHSG) HD9 trial showed a significant improvement in both disease control (time-to-treatment-failure) and overall survival (OS) for patients treated upfront with standard-dose BEACOPP or eBEACOPP, compared with alternating cycles of COPP (cyclophosphamide, vincristine, procarbazine, and prednisone) and ABVD [1]. Subsequently, eBEACOPP was introduced as a treatment option at six cancer centres in the Anglia Cancer Network (ACN) for certain patients for whom it was considered clinically appropriate, whilst two other ACN centres continued to offer ABVD for all patients. From 2008 to 2012, four of the ACN centres also recruited patients onto the Response-Adjusted Therapy for Advanced Hodgkin Lymphoma (RATHL) trial. In RATHL, all patients received two cycles of ABVD followed by an interim positronemission tomography-computed tomography (PET/CT) scan (iPET2) to guide further treatment intensity. Patients with a negative iPET2 scan (Deauville score 1-3) were randomised 1:1 to receive a further four cycles of ABVD or AVD (without bleomycin). Patients with a positive iPET2 scan (Deauville score 4-5) had their treatment intensified and received either a further three cycles of eBEACOPP or four cycles of BEACOPP-14 (standard-dose BEACOPP delivered every 14 days), without randomisation. Radiotherapy (RT) was not mandated in the trial protocol but could be offered at the discretion of the treating clinician. The 3-year follow-up results of RATHL have been published [2].

In our retrospective study, we looked specifically at young patients, aged 16-59 years, with advanced-stage HL who were treated with first-line ABVD or eBEACOPP in the ACN over a 10-year period (2004-2014), which included the 5 years immediately preceding and following the introduction of eBEACOPP at six ACN centres in 2009. We compared our outcomes against an updated 5-year analysis of outcomes for RATHL patients, aged 18-59 years. This allowed us to compare progression-free survival (PFS) and OS for patients treated upfront with ABVD or eBEACOPP in a non-trial (socalled real-world), multicentre setting, against patients treated in a large, contemporary ABVD-based prospective trial that incorporated interim-PET/CT-guided dose-escalation for patients with an inferior response after two cycles of standard ABVD induction therapy.

\section{Methods}

\section{Data collection}

We collected data retrospectively from eight cancer centres in the Anglia Cancer Network (ACN), including Cambridge University Hospital and Nuffield Hospital, Cambridge; Norfolk and Norwich University Hospital, Norwich; Ipswich Hospital, Ipswich; James Paget University Hospital, Great Yarmouth; Hinchingbrooke Hospital, Hinchingbrooke; Peterborough City Hospital, Peterborough; Queen Elizabeth Hospital, King's Lynn; and West Suffolk Hospital, Bury Saint Edmunds. Eligible patients were identified by review of electronic and paper hospital records, local cancer registry data, and the NHS database. Study inclusion criteria included all young (aged 16-59 years), treatment-naïve patients diagnosed with advanced-stage HL (stage IIB-IV, or IIA with bulk disease defined as $>0.33$ transthoracic diameter or $>10 \mathrm{~cm}$ ) in the ACN between July 1, 2004, and June 31, 2014, who were treated outside of a clinical trial (so-called real-world) with either upfront ABVD or eBEACOPP. To provide comparable durations of follow-up, real-world ABVD and eBEACOPP patients were censored in April 2016 and January 2019, respectively, and RATHL trial patients were censored in June 2019.

\section{Statistical analysis}

Statistical analysis was conducted using Stata version 16.1 software (StataCorp, TX, USA). Baseline variables of interest were calculated using standard summary statistics and included age at diagnosis, sex, biopsy date, Ann Arbor stage, international prognostic score (IPS), treatment regimen, use of radiotherapy, date of relapse and/or death. Pearson's chisquared test for categorical variables was applied for the comparison between groups. Survival analysis was calculated 
from the date of diagnostic biopsy to the date of first progression or death from all causes. Surviving patients without progression were censored at the date they were last known to be alive. Survival estimates with $95 \%$ confidence intervals were calculated using the Kaplan-Meier method and compared using the log-rank test. Hazard ratios (HR) with $95 \%$ confidence intervals were calculated using a Cox regression model. A $p$-value of $<0.05$ was considered significant. Median follow-up duration was calculated using the reverse KaplanMeier method, and was censored at death from all causes. Statistical analysis of the 5-year follow-up data from the RATHL trial for patients aged 18-59 years, and subsequent comparative analysis with our real-world ACN cohort, was performed by the RATHL trial team.

\section{Results}

We identified 250 patients, aged $16-59$ years, diagnosed with advanced-stage HL in the ACN from July 1, 2004, to June 31, 2014. This number is in keeping with the expected incidence for the ACN population of 2.64 million [3]. Of these 250 patients, 29 were excluded, including 25 who were treated in the RATHL trial, three who were treated with regimens other than ABVD or eBEACOPP, and one who died before commencing therapy. There were 221 patients eligible for study inclusion, with a median follow-up of 87 months. A flowchart of the treatment pathway for the whole cohort is shown in Fig. 1. Real-world patient outcomes were compared with 5-year outcomes for patients treated in the RATHL trial, aged 18-59 years $(n=1088)$, with a median follow-up of 69.5 months.

Comparison of the baseline characteristics of real-world and RATHL patients showed highly similar age and sex distribution, but the real-world cohort had significantly fewer stage II patients and significantly more stage IV $(p<0.001)$ and higher risk (IPS $3+$ ) patients $(p=<0.001$ ), compared with the RATHL trial (Table 1). In the real-world cohort, there was a patient-clinician bias to treat higher-risk patients with moreintensive induction therapy, with $75 \%$ of eBEACOPP patients being IPS $3+$ compared with only $39 \%$ of ABVD patients $(p<0.001)$

The 5-year PFS and OS estimates for the whole cohort of real-world patients $(n=221)$ were $82.5 \%$ and $93.9 \%$, respectively. These results were highly similar for RATHL patients aged $18-59$ years $(n=1088)$; PFS $81.4 \%$; HR $1.05, p=0.79$; OS $95.2 \%, p=0.71$; Fig. 2 Of the 177 real-world ABVD patients, the majority $(n=130 ; 73.4 \%)$ completed six cycles of ABVD, and 41 (23.2\%) completed eight cycles. Interim-PET/ CT scans were not routinely performed in the real-world setting. Five patients $(2.8 \%)$ had their treatment intensified to eBEACOPP after two, three, or four cycles of ABVD, and two patients $(1.1 \%)$ were de-escalated to ChlVPP (chlorambucil, vinblastine, procarbazine, and prednisolone) after three cycles of ABVD. Bleomycin was stopped after two or three cycles in 15 patients $(8.5 \%)$ because of pulmonary toxicity. Twenty patients of the ABVD cohort (11\%) received consolidative radiotherapy (RT) after completing frontline chemotherapy, including 16/20 who were treated exclusively with ABVD therapy (15/16 received RT to areas of residual bulk and 1/16 for progressive disease), $3 / 20$ who had their treatment intensified to eBEACOPP $(3 / 3$ received IFRT to areas of residual bulk), and 1/20 who had their treatment de-escalated to ChlVPP). By comparison, 154 (14\%) RATHL patients had their treatment intensified to eBEACOPP or BEACOPP-14 after two cycles of ABVD as per trial protocol, and $73(6.7 \%)$ patients received consolidative RT [2].

Of the 44 real-world eBEACOPP patients, 31 (70.5\%) were treated exclusively with eBEACOPP, of which the

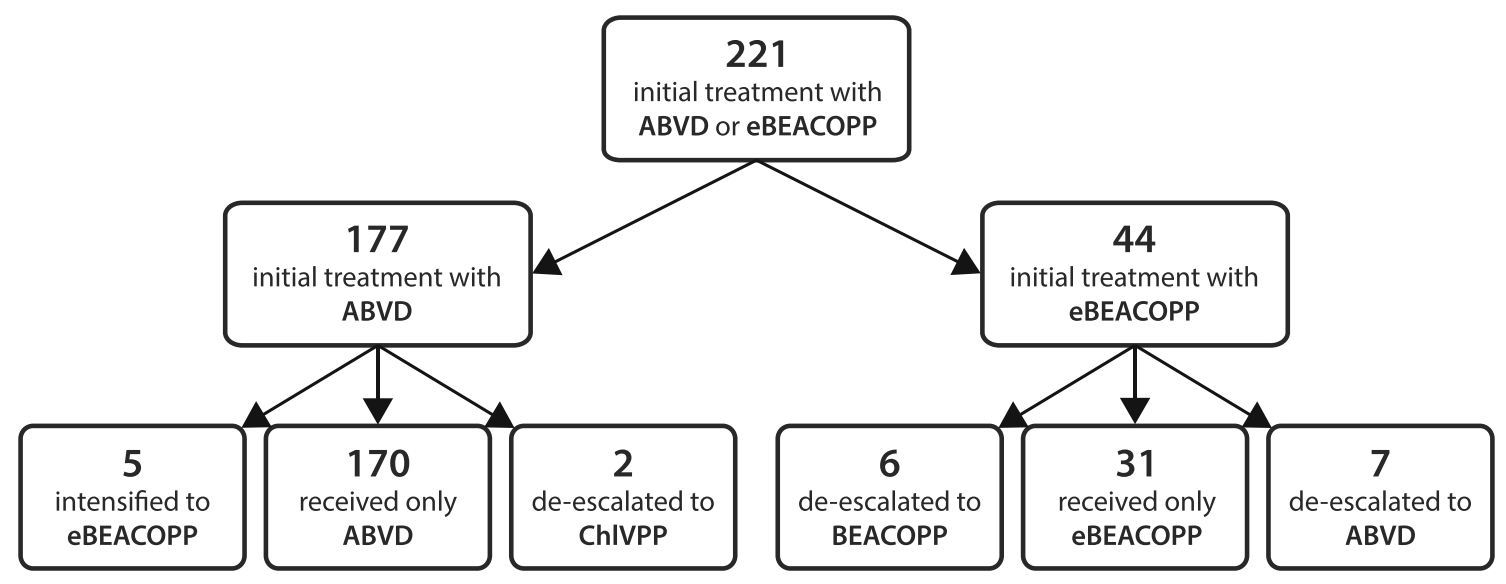

Fig. 1 Flowchart of the treatment pathway for 221 young advanced-stage classical Hodgkin lymphoma patients diagnosed in the Anglia Cancer Network, from 2004 to 2014. ABVD, doxorubicin, vinblastine, bleomycin, and dacarbazine; BEACOPP, bleomycin, etoposide,

doxorubicin, cyclophosphamide, vincristine, procarbazine, and prednisolone; ChlVPP, chlorambucil, vinblastine, procarbazine, and prednisolone; eBEACOPP, escalated-BEACOPP 
Table 1 Baseline characteristics of 221 young advanced-stage Hodgkin lymphoma patients treated in the real-world (RW) setting compared with 1088 patients treated in the RATHL trial. $A B V D$, doxorubicin, vinblastine, bleomycin, and dacarbazine; $e B E A C O P P$, escalated-bleomycin, etoposide, doxorubicin, cyclophosphamide, vincristine, procarbazine, and prednisolone; RATHL, responseadapted therapy for advanced Hodgkin lymphoma

\begin{tabular}{|c|c|c|}
\hline Baseline characteristics & Real-world, $N=221$ & RATHL, $N=1088$ \\
\hline Median age at diagnosis - y (range) & $35(16-59)$ & $31(18-59)$ \\
\hline Male sex- $n(\%)$ & $121(54.8)$ & $585(53.8)$ \\
\hline \multicolumn{3}{|l|}{ Median follow-up-mo. $(95 \% \mathrm{CI})$} \\
\hline Whole cohort & $87.0(83.0-94.0)$ & $69.5(67.2-69.5)$ \\
\hline ABVD & $87.0(83.0-94.3)$ & - \\
\hline eBEACOPP & $88.0(79.3-102.1)$ & - \\
\hline \multicolumn{3}{|l|}{ Ann Arbor stage $-n(\%)$} \\
\hline II with adverse features & $62(28.1)$ & $471(43.3)$ \\
\hline III & $68(30.8)$ & $312(28.7)$ \\
\hline IV & $91(41.2)$ & $305(28.0)$ \\
\hline \multicolumn{3}{|l|}{ International Prognostic Score $-n(\%)$} \\
\hline $0-2$ & $117(52.9)$ & $728(67.0)$ \\
\hline $3-6$ & $102(46.2)$ & $359(33.0)$ \\
\hline Unknown & $2(0.9)$ & $1(0.0)$ \\
\hline \multicolumn{3}{|l|}{ Initial treatment $-n(\%)$} \\
\hline ABVD & $177(80.1)$ & $1088(100.0)$ \\
\hline eBEACOPP & $44(19.9)$ & $0(0.0)$ \\
\hline
\end{tabular}

majority $(n=25 ; 80.6 \%)$ completed six cycles, four $(12.9 \%)$ completed five cycles, one $(3.2 \%)$ completed seven cycles, and one $(3.2 \%)$ completed eight cycles. Six patients $(13.6 \%)$ were de-escalated to standard-dose BEACOPP after either two $(n=3,6.8 \%)$, three $(n=1,2.3 \%)$, or four $(n=2,4.5 \%)$ cycles of eBEACOPP. Seven patients $(15.9 \%)$ were deescalated to ABVD after one $(n=1,2.3 \%)$, two $(n=4,9.1 \%)$, three $(n=1,2.3 \%)$, or four $(n=1,2.3 \%)$ cycles of eBEACOPP. De-escalation was primarily required because of toxicity; however, three patients requested de-escalation due to fertility concerns. Of the 44 eBEACOPP patients, 5 patients $(11 \%)$ received consolidative $\mathrm{RT}$ to areas of residual nodal tissue $>$ $2 \mathrm{~cm}$ with residual metabolic uptake on PET/CT. No patients in this cohort had RT for primary refractory disease. Kaplan-

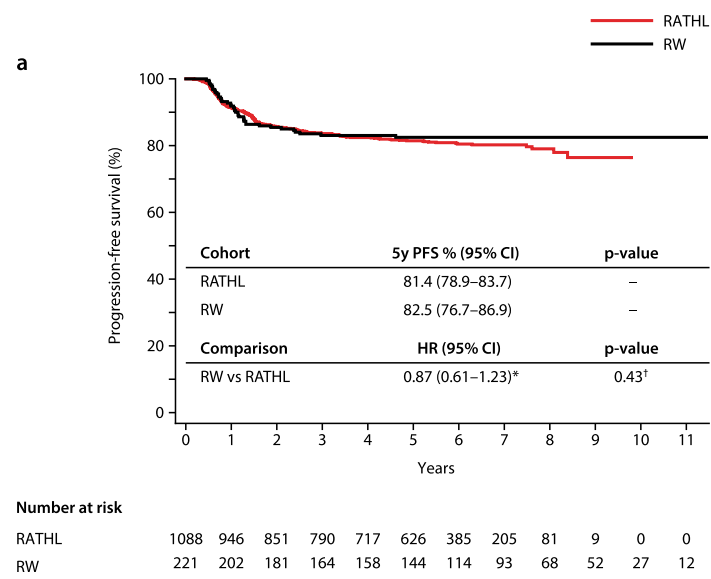

Fig. 2 Kaplan-Meier curves of survival estimates for the whole cohort of real-world (RW) patients compared with RATHL trial patients. a Progression-free survival (PFS). b Overall survival (OS). HR, hazard
Meier curves of PFS and OS for real-world patients, by treatment regimen, and RATHL trial patients are shown in Fig. 3.

The 5-year PFS and OS estimates for real-world ABVD patients $(n=177)$ were $79.2 \%$ and $92.9 \%$ respectively. Survival outcomes were highly similar in RATHL patients, aged 18-59 years; PFS 81.4\%, HR 1.05, $p=0.79$; OS 95.2\%, $p=0.71$. Although the real-world eBEACOPP cohort comprised proportionately more higher-risk patients (75\% were IPS $3+$, compared with $39 \%$ of real-world ABVD patients and $33 \%$ of RATHL patients), it had a statistically significant PFS advantage compared with the real-world ABVD cohort (HR 0.20, $p=0.027$ ) and RATHL trial (HR 0.21, $p=0.015$; Fig. $3 \mathrm{a}$ ). However, there was no statistically significant OS advantage (Fig. 3b).

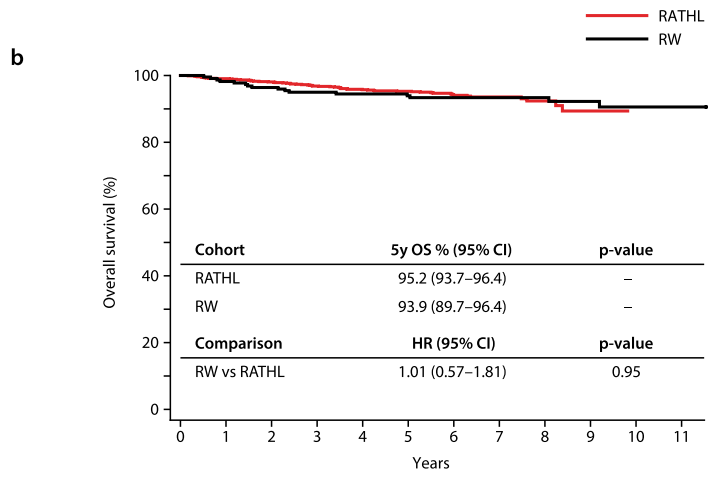

Number at risk

$\begin{array}{lllllllllllll}\text { RATHL } & 1088 & 1020 & 971 & 912 & 834 & 728 & 454 & 237 & 92 & 11 & 0 & 0\end{array}$ $\begin{array}{lllllllllllll}\text { RW } & 221 & 217 & 207 & 188 & 180 & 169 & 139 & 114 & 85 & 62 & 32 & 13\end{array}$

ratio; RATHL, response-adapted therapy for advanced Hodgkin lymphoma; $\dagger \log$ rank $p$-value; *fails the assumption of proportional hazards 


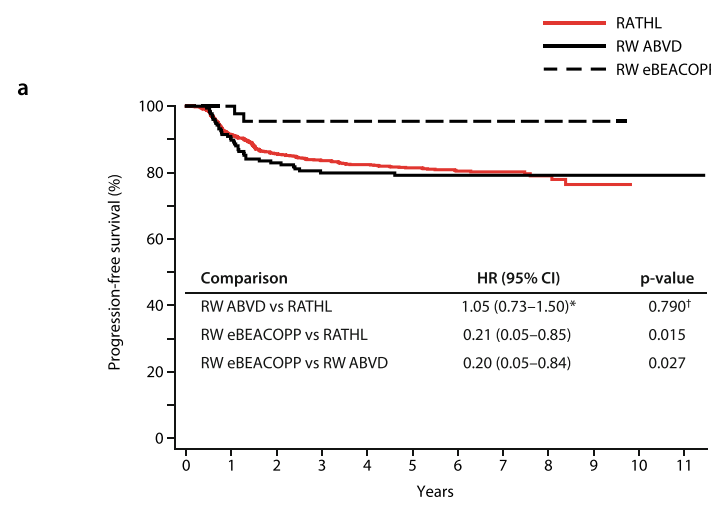

Number at risk

RATHL

RW ABVD

RW EBEACOPP

Fig. 3 Kaplan-Meier curves of survival estimates for real-world (RW) patients, grouped by treatment regimen, compared with RATHL trial patients. a Progression-free survival (PFS). b Overall survival (OS). ABVD, doxorubicin, vinblastine, bleomycin, and dacarbazine; eBEACOPP, escalated-bleomycin, etoposide, doxorubicin,

With the RATHL trial, a higher IPS was associated with a poorer response to ABVD induction therapy, with $22.1 \%$ of IPS 3+ patients being iPET2-positive versus $12.9 \%$ of IPS 0-2 patients. With ongoing follow-up, IPS remains prognostic of 5-year PFS (HR 1.61, $p=0.0008$ ) despite a greater proportion of IPS $3+$ patients receiving treatment intensification (Fig. 4a).

Real-world IPS 3+ eBEACOPP patients had significantly improved 5-year PFS and OS estimates compared with real-world IPS 3+ ABVD patients (PFS 97.0\% vs $71.9 \%$; HR $0.09, p=0.021$, Fig. $4 \mathrm{~b}$; OS $100 \%$ vs $84.5 \%$, $p=0.0449$, Fig. 4c). In addition, real-world IPS $3+$ eBEACOPP patients retained a significant PFS benefit compared with IPS $3+$ RATHL patients $(97.0 \%$ vs $76.3 \%$; HR $0.10, p=0.005$; Fig. 4 b) despite $22.1 \%$ of IPS 3+ RATHL patients receiving treatment intensification after two cycles of ABVD, as per protocol. Although a higher OS rate was observed for the real-world IPS 3+ eBEACOPP patients compared to the IPS 3+ RATHL patients (100\% vs 91.4\%; HR 0.27), this did not reach statistical significance $(p=0.16)$ (Fig. $4 \mathrm{c})$.

The 5-year survival estimates for real-world and RATHL trial patients are presented for all patient subgroups in Table 2 .

\section{Fertility}

Seventeen pre-menopausal women were treated with upfront eBEACOPP in our real-world cohort, of which 13 $(76.5 \%)$ were younger than 30 years at diagnosis. Following completion of treatment, $12(70.6 \%)$ regained menstrual periods (11 of whom were younger than 30 years at diagnosis), four (23.5\%) were diagnosed with premature ovarian failure, and one $(5.9 \%)$ died from complications related to allogeneic stem cell transplantation.

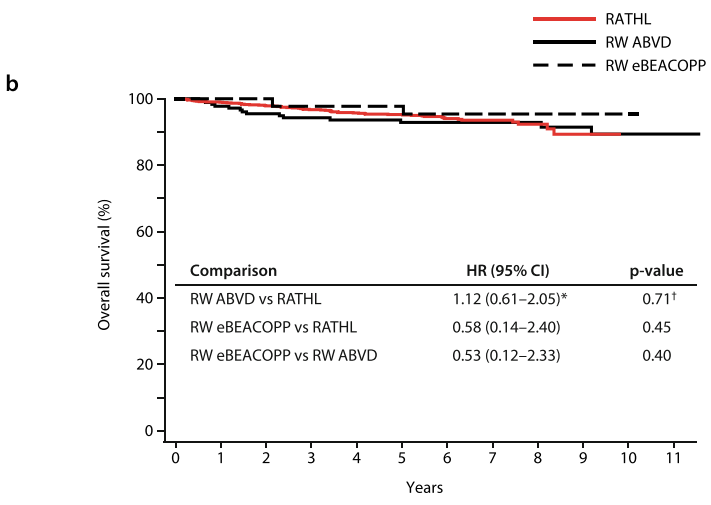

$\begin{array}{lcccccccccccc}\text { Number at risk } & & & & & & & & & & & & \\ \text { RATHL } & 1088 & 1020 & 971 & 912 & 834 & 728 & 454 & 237 & 92 & 11 & 0 & 0 \\ \text { RW ABVD } & 177 & 173 & 163 & 145 & 137 & 127 & 106 & 92 & 68 & 51 & 31 & 13 \\ \text { RW eBEACOPP } & 44 & 44 & 44 & 43 & 43 & 42 & 33 & 22 & 17 & 11 & 1 & 0\end{array}$

cyclophosphamide, vincristine, procarbazine, and prednisolone; HR, hazard ratio; RATHL, response-adapted therapy for advanced Hodgkin lymphoma; †log rank $p$-value; *fails the assumption of proportional hazards

At the time of data collection, five of these 17 women (29.4\%) had had a total of seven pregnancies, which resulted in five live-births and two terminations of pregnancy.

\section{Use of stem cell transplantation}

Figure 5 shows a flowchart of real-world patients treated with stem cell transplantation (SCT) for relapsed/ refractory $\mathrm{HL}$ or second malignancies, after frontline therapy with ABVD or eBEACOPP. There was a statistically significant difference in the rate of autologous SCT (autograft) between the real-world ABVD and eBEACOPP groups $(p=0.0415)$. In the real-world ABVD cohort, 26 patients (14.7\%) received an autograft; in 25 patients, this was after salvage therapy for relapsed HL, and in one patient as treatment of T-cell lymphoma. Three real-world ABVD patients (1.7\%) had an allogeneic SCT (allograft) for relapsed HL, including one patient who had previously had an autograft.

In the real-world eBEACOPP group, one patient $(2.3 \%)$ had an autograft after salvage therapy for relapsed HL, followed by an allograft after a second relapse. A further two eBEACOPP patients had an allograft, both without previous autograft, including one patient with relapsed HL who did not mobilise sufficient stem cells for an autograft after salvage chemotherapy and who received an allograft after a second relapse, and one patient with therapy-related myelodysplastic syndrome (t-MDS), comprising a total of three eBEACOPP patients $(6.8 \%)$ who received an allograft compared with $1.7 \%$ in the ABVD group. 


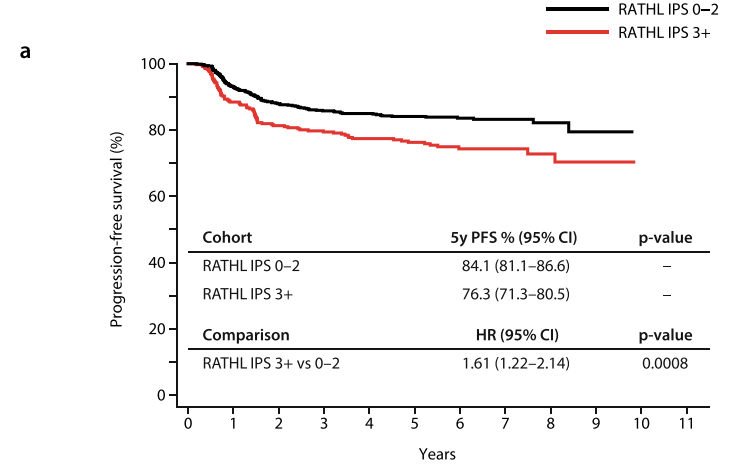

Number at risk

$\begin{array}{lllllllllllll}\text { RATHL IPS 0-2 } & 726 & 643 & 582 & 544 & 492 & 436 & 266 & 136 & 50 & 7 & 0 & 0\end{array}$ $\begin{array}{lllllllllllll}\text { RATHLIPS 3+ } & 359 & 301 & 267 & 244 & 225 & 190 & 119 & 69 & 31 & 2 & 0 & 0\end{array}$

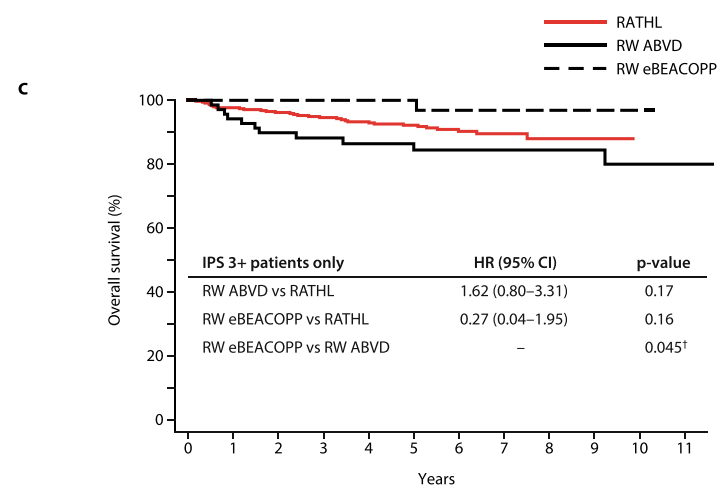

Number at risk

$\begin{array}{lllllllllllll}\text { RATHLIPS 3+ } & 359 & 330 & 315 & 291 & 273 & 229 & 147 & 81 & 35 & 3 & 0 & 0\end{array}$ $\begin{array}{llllllllllllll}\text { RW ABVD IPS 3+ } & 69 & 65 & 59 & 50 & 48 & 43 & 38 & 34 & 27 & 22 & 14 & 6\end{array}$ $\begin{array}{lllllllllllll}\text { RW EBEACOPP IPS 3+ } & 33 & 33 & 33 & 33 & 33 & 32 & 27 & 18 & 14 & 11 & 1 & 0\end{array}$

Fig. 4 Kaplan-Meier curves of survival estimates for real-world (RW) and RATHL trial patients, grouped by treatment regimen and international prognostic score (IPS). a Progression-free survival (PFS) for RATHL trial patients, grouped by IPS. b PFS for IPS 3+ real-world patients, grouped by treatment regimen, compared with IPS 3+ RATHL trial patients. c Overall survival (OS) for IPS 3+ real-world patients, grouped by treatment regimen, compared with IPS 3+ RATHL trial

\section{Discussion}

ABVD and eBEACOPP remain the most widely used firstline treatments of advanced-stage HL, and are recommended treatment options in both the current UK and European Society for Medical Oncology (ESMO) guidelines [4, 5]. It is broadly accepted that eBEACOPP offers more effective anti-Hodgkin therapy, but at the cost of additional toxicity. As such, it remains a case-by-case decision as to which regimen is more appropriate for a given patient.

In this retrospective study, we compared the outcomes of patients treated with upfront ABVD or eBEACOPP in a multicentre real-world setting, against patients treated in the RATHL trial. A strength of this study is the multi-hospital real-world dataset that has been rigorously checked to include all age-appropriate patients treated for Hodgkin lymphoma in the cancer network over a 10-year period. However, there are

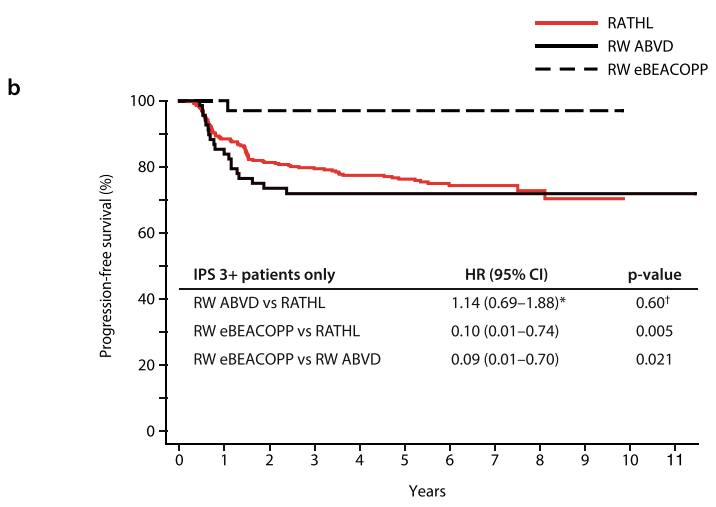

Number at risk

$\begin{array}{lllllllllllll}\text { RATHLIPS 3+ } & 359 & 301 & 267 & 244 & 225 & 190 & 119 & 69 & 31 & 2 & 0 & 0\end{array}$ $\begin{array}{lllllllllllll}\text { RW ABVD IPS 3+ } & 69 & 57 & 47 & 42 & 42 & 38 & 34 & 29 & 22 & 18 & 11 & 5\end{array}$ $\begin{array}{lllllllllllll}\text { RW eBEACOPP IPS 3+ } & 33 & 33 & 31 & 30 & 29 & 25 & 20 & 14 & 11 & 8 & 0 & 0\end{array}$

patients. $\mathrm{ABVD}$, doxorubicin, vinblastine, bleomycin, and dacarbazine; eBEACOPP, escalated-bleomycin, etoposide, doxorubicin, cyclophosphamide, vincristine, procarbazine, and prednisolone; HR, hazard ratio; RATHL, response-adapted therapy for advanced Hodgkin lymphoma; $\dagger \log$ rank $p$-value; $*$ fails the assumption of proportional hazards

inherent limitations with real-world retrospective case studies which include treatment selection bias and particularly with our study, the relatively small number of patients and events in the eBEACOPP treatment cohort which inevitably introduces more uncertainty in the subsequent comparative statistical analysis. The over-representation of stage II patients in RATHL compared with other large prospective Hodgkin lymphoma trials has been the subject of previous discussion [6], but despite this limitation, it is the largest contemporary multicentre trial evaluating ABVD in the modern era. A similar but smaller trial was run by the US SWOG group (S0816) where iPET-positive patients were also intensified to eBEACOPP. In contrast with RATHL, however, only stage III/IV patients were included and dose-intensified patients received 6 rather than 4 cycles of eBEACOPP. The overall 5year PFS for S0816 was $74 \%$ which appears inferior to RATHL, possibly reflecting the inclusion criteria for the trial. 
Table 2 Summary of 5-year survival estimates for real-world (RW) and RATHL trial patients, grouped by treatment regimen, clinical stage, and international prognostic score (IPS). $A B V D$, doxorubicin, vinblastine, bleomycin, and dacarbazine; $e B E A C O P P$, escalated-bleomycin, etoposide, doxorubicin, cyclophosphamide, vincristine, procarbazine, and prednisolone; $H R$, hazard ratio; $R A T H L$, response-adapted therapy for advanced Hodgkin lymphoma; *fails the assumption of proportional hazards

\begin{tabular}{|c|c|c|c|c|}
\hline Endpoint/group & $\begin{array}{l}\mathrm{RW} \text { ABVD } \\
5 \text {-year rate } \%(n ; 95 \% \mathrm{CI})\end{array}$ & $\begin{array}{l}\text { RW eBEACOPP } \\
5 \text {-year rate } \%(n ; 95 \% \mathrm{CI})\end{array}$ & $\begin{array}{l}\text { RATHL } \\
5 \text {-year rate } \%(n ; 95 \% \mathrm{CI})\end{array}$ & $\begin{array}{l}\text { RW ABVD vs RATHL } \\
\text { HR ( } 95 \% \text { CI); } p \text {-value }\end{array}$ \\
\hline \multicolumn{5}{|c|}{ Progression-free survival } \\
\hline Whole cohort & $79.2(177 ; 72.3-84.5)$ & $95.5(44 ; 83.0-98.8)$ & $81.4(1088 ; 78.9-83.7)$ & $1.05(0.73-1.50) ; 0.79$ \\
\hline IPS 0-2 & $84.7(106 ; 76.2-90.3)$ & $90.9(11 ; 50.8-98.7)$ & $84.1(728 ; 81.1-86.6)$ & $0.89(0.52-1.50) ; 0.66$ \\
\hline IPS $3+$ & $71.9(69 ; 59.5-81.1)$ & $97.0(33 ; 80.4-99.6)$ & $76.3(359 ; 71.3-80.5)$ & $1.14 *(0.69-1.88) ; 0.60$ \\
\hline Stage IV & $73.6(59 ; 60.0-83.2)$ & $96.9(32 ; 79.8-99.6)$ & $77.0(305 ; 71.6-81.5)$ & 1.04 (0.59-1.82); 0.90 \\
\hline \multicolumn{5}{|l|}{ Overall survival } \\
\hline Whole cohort & $92.9(177 ; 87.8-95.9)$ & $97.7(44 ; 84.9-99.7)$ & $95.2(1088 ; 93.7-96.4)$ & $1.12 *(0.61-2.05) ; 0.71$ \\
\hline IPS $0-2$ & $98.1(106 ; 92.5-99.5)$ & $90.9(11 ; 50.8-98.7)$ & $96.7(728 ; 95.0-97.9)$ & 0.47 (0.14-1.59); 0.22 \\
\hline IPS $3+$ & $84.5(69 ; 72.9-91.4)$ & $100(33 ; \mathrm{N} / \mathrm{A})$ & $92.2(359 ; 88.7-94.7)$ & $1.62(0.80-3.31) ; 0.18$ \\
\hline Stage IV & $92.6(59 ; 81.3-97.2)$ & $100(32 ; \mathrm{N} / \mathrm{A})$ & $91.5(305 ; 87.4-94.3)$ & $0.82(0.31-2.16) ; 0.69$ \\
\hline
\end{tabular}

Both RATHL and SWOG S0816 reflect the modern practice of using minimal consolidative RT and we felt RATHL was an appropriate benchmark for comparison with our real-world dataset. The PFS curves of the real-world ABVD patients and RATHL trial patients, aged younger than 60 years, are remarkably similar in both the absolute 5-year estimates of progression-free survival and in the kinetics of relapse. This is observed in both the whole ABVD cohort and IPS 3+ subgroup, and is consistent with data published from the Netherlands that suggest similar outcomes are achieved for
HL patients who are treated within or outside of clinical trials [7].

It is clear from RATHL that higher-risk (IPS 3+) patients are more likely to be iPET2 positive after ABVD induction therapy, and despite treatment intensification with eBEACOPP or BEACOPP-14, they also had a higher relapse rate $(32.5 \%)$ at 3 years [2]. Whilst, intuitively, it seems appropriate to intensify therapy in iPET2-positive patients, this has not been tested in a randomised trial and the magnitude of benefit from this strategy is unknown. Notably, only $2.6 \%$

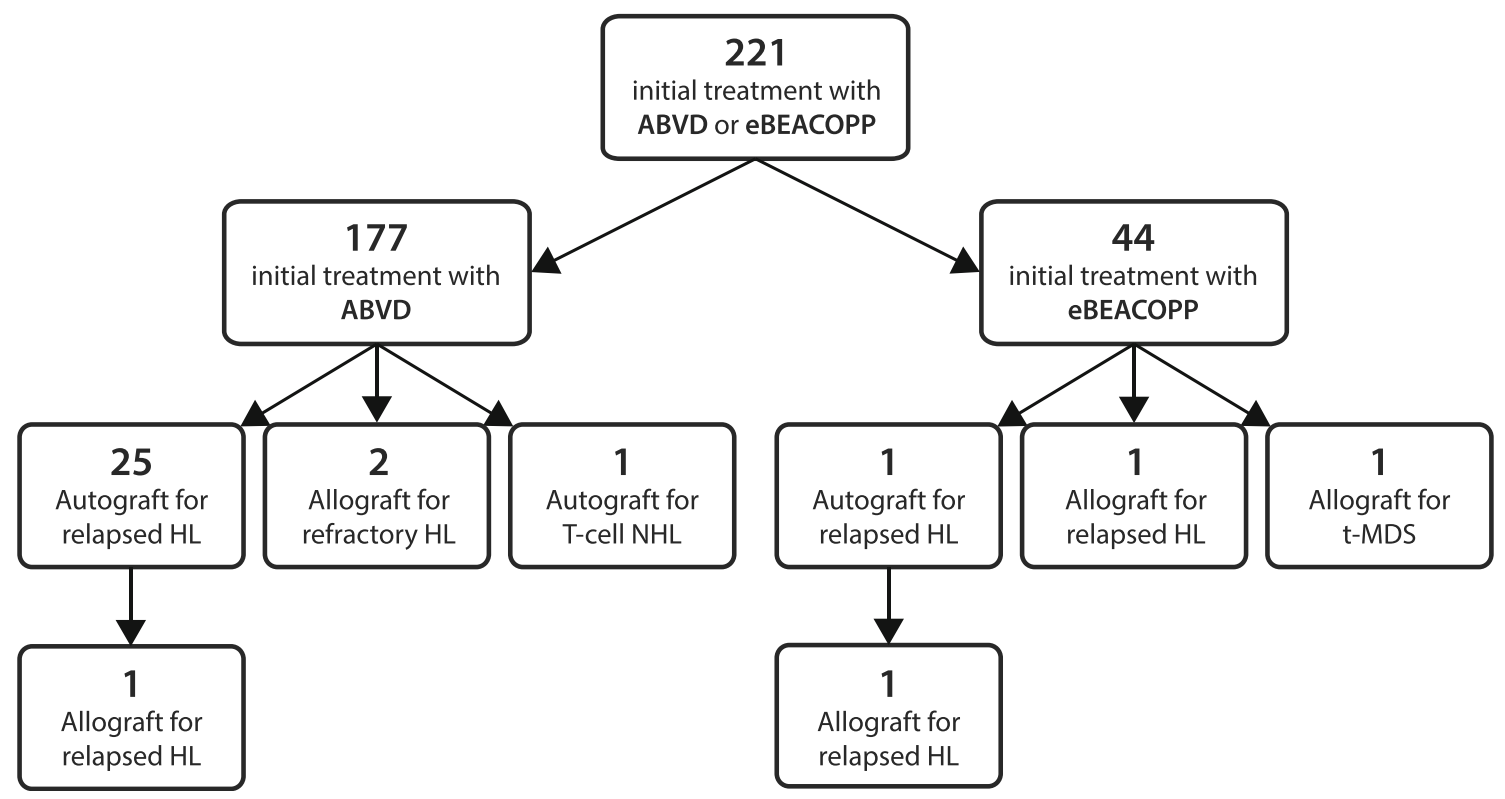

Fig. 5 Flowchart of real-world patients treated with autologous (autograft) and/or allogeneic (allograft) stem cell transplantation for relapsed/refractory classical Hodgkin lymphoma (HL) or second malignancies, grouped by initial treatment regimen. ABVD,

doxorubicin, vinblastine, bleomycin, and dacarbazine; eBEACOPP, escalated-bleomycin, etoposide, doxorubicin, cyclophosphamide, vincristine, procarbazine, and prednisolone; NHL, non-Hodgkin lymphoma; t-MDS, therapy-related myelodysplastic syndrome 
of real-world ABVD patients had treatment intensification to eBEACOPP after induction ABVD, compared with $16.1 \%$ of RATHL patients, yet there was no observed difference in PFS between the real-world ABVD and RATHL patients in either the whole ABVD cohort or IPS 3+ subgroup. This suggests that either the magnitude of benefit for treatment intensification based on an iPET2 scan is limited or that there are unknown competing factors influencing the data.

In both our study and clinical trials, it seems that higherrisk patients achieve the greatest relative gain in PFS when treated with upfront eBEACOPP compared with ABVD. The RATHL trial has shown that even with interim-PET/CT-directed treatment intensification, IPS $3+$ patients have an inferior PFS compared with IPS 0-2 patients. This is not solely explained by fewer patients achieving an iPET2-negative remission, as those IPS 3+ patients who achieved an iPET2negative remission had a higher risk of relapse compared with IPS $0-2$ patients $(18.3 \%$ vs $13.1 \%$ at 3 years, HR 1.44 [1.042.01], $p=0.029$ ) [2] (Kirkwood, A, personal communication, 2016)

Although there has never been a randomised prospective trial that compared six cycles of eBEACOPP with six cycles of $A B V D$, randomised trials that have compared variations of these regimens have consistently shown a first-remission PFS advantage with upfront eBEACOPP, ranging from 5 to $18 \%$ [8-10]. The 5-year PFS difference between patients treated with upfront eBEACOPP compared with ABVD in our unselected real-world cohort was $16 \%$. In keeping with prospective trial data, OS estimates were excellent in both of our treatment groups, suggesting that patients who relapse are frequently salvaged, albeit with the need for more toxic regimens. The comparative infrequency of relapse in real-world eBEACOPP patients correlates with their significantly lower likelihood of receiving an autologous stem cell transplant, compared with ABVD patients. Although not planned prospectively, there was clear clinician bias to treat IPS $3+$ patients with upfront eBEACOPP in those centres where it was available. Notably, IPS 3+ eBEACOPP patients were also observed to have a disproportionate PFS benefit compared with IPS 3+ ABVD patients.

With regard to OS, IPS 3+ real-world eBEACOPP patients had an OS advantage compared with IPS 3+ real-world ABVD patients and showed a difference of just over $8 \%$ in 5-year OS when compared with IPS 3+ RATHL patients. Although this did not reach statistical significance, this may be due to the small number of patients and events, particularly within the real-world cohort ( 1 event in 33 patients). This difference is not that dissimilar to differences shown in meta-analyses comparing upfront ABVD to BEACOPP [11] which saw 5-year OS differences of $7 \%$ between ABVD $(88 \%$ 5 -year OS) and 6 cycles of eBEACOPP (95\%).

As treatments for first line and relapsed Hodgkin lymphoma evolve and more options become available for our patients, the number of younger adults dying from Hodgkin lymphoma is thankfully decreasing. However, all treatment options at relapse bring more toxicities for patients, particularly when transplant strategies are employed, and achieving a durable first remission remains a highly important goal for our patients. With the publication of the HD18 and AHL2011 trials $[12,13]$, the cumulative toxicity of eBEACOPP strategies has been reduced with no impact on PFS, making the eBEACOPP strategy even more appealing as a first-line treatment option for advanced stage HL. Indeed, with longer follow-up of HD18, patients who achieved an interim metabolic remission after 2 cycles of eBEACOPP had an improved overall survival when treated with a total of 4 cycles of eBEACOPP compared with 6-8 cycles, owing to a reduction in treatment-related mortality [14]. This confirms that PETguided strategies can optimise the PFS and OS for the majority of eBEACOPP-treated patients, whilst reducing the overall toxicity of the regimen.

Reduced fertility is a significant concern for patients treated with eBEACOPP. We reviewed the outcomes for menstrual cycle recovery and fertility after eBEACOPP and found consistent premature ovarian failure in pre-menopausal women aged over 30 years at diagnosis $(n=4)$, with only one patient having a transient return of menstrual periods. Of the 13 premenopausal women treated with eBEACOPP aged under 30 years at diagnosis, the majority $(n=11,84.6 \%)$ regained menstrual periods and several pregnancies have been carried to term. Using anti-mullerian hormone levels as a surrogate for fertility, prospective follow-up of female patients from the RATHL study has confirmed that age over 35 and treatment with BEACOPP were both independent predictors of reduced AMH levels post-treatment, whilst the majority of woman under 35 treated with ABVD/AVD regained pre-treatment AMH levels by 2 years post-treatment [15]. Reducing the number of cycles of eBEACOPP is likely to help preserve fertility. Prospective trials that use interim-PET/CT assessment to de-escalate the better-risk patients after eBEACOPP induction therapy, such as in the recently published LYSA AHL2011, and HD18 trials, have shown encouraging results $[12,16]$. It is now general practice in the UK to offer pretreatment fertility counselling, including potential oocyte storage, to all women of reproductive age when clinically appropriate.

Second primary malignancies (SPM) are another concern for patients receiving first-line therapy for Hodgkin lymphoma and relative risks remain elevated for patients compared with age-matched controls whether treated with chemotherapy alone or in combination with radiotherapy. Meta-analysis of large trial datasets suggests an increased risk of therapyrelated acute myeloid leukemia/myelodysplastic syndromes in patients treated with dose-intensified therapy [12]. However, as seen with our real-world dataset, patients treated with ABVD are more likely to need dose intensification after 
an interim PET scan or at relapse, so it remains difficult to advise patients of their individual long-term risk of a SPM when first-line therapy choices are made. With the RATHL patients to date, 4 non-cutaneous SPMs have been diagnosed in 154 patients who were dose escalated to BEACOPP-based chemotherapy (Kirkwood, A; personal communication).

In conclusion, our data provide further evidence that the outcomes for frontline treatment of young adults (aged younger than 60 years) with advanced-stage HL in a real-world setting are highly similar to those achieved in prospective clinical trials. Although RATHL-style intensification of therapy for iPET2-positive patients has become an accepted standard of care in the UK, 5-year PFS estimates for RATHL patients, aged 18-59 years, were highly similar to patients treated in a multicentre real-world setting with no iPET2guided intensification strategy. We observed a clear PFS benefit for patients treated with upfront eBEACOPP and recent UK data have shown that since the publication of HD18, there has been a marked increase in the number of UK centres using eBEACOPP/eBEACOPDacarbazine as first-line therapy to treat advanced stage HL [17].

Data availability Due to privacy and ethical concerns, the real-world data analysed in this study is not publicly available.

\section{Code availability Not applicable.}

\section{Declarations}

Ethics approval Ethics board approval was not required in view of the retrospective nature of the study and all the interventions being performed were part of routine care.

Consent to participate Consent to participate was not required for this real-world patient cohort as the study involved a retrospective chart review of anonymous patient data.

Consent for publication Consent for publication was not required for this real-world patient cohort as the study involved a retrospective chart review of anonymous patient data.

Conflict of interest The authors declare no competing interests.

Open Access This article is licensed under a Creative Commons Attribution 4.0 International License, which permits use, sharing, adaptation, distribution and reproduction in any medium or format, as long as you give appropriate credit to the original author(s) and the source, provide a link to the Creative Commons licence, and indicate if changes were made. The images or other third party material in this article are included in the article's Creative Commons licence, unless indicated otherwise in a credit line to the material. If material is not included in the article's Creative Commons licence and your intended use is not permitted by statutory regulation or exceeds the permitted use, you will need to obtain permission directly from the copyright holder. To view a copy of this licence, visit http://creativecommons.org/licenses/by/4.0/.

\section{References}

1. Engert A, Diehl V, Franklin J, Lohri A, Dörken B, Ludwig WD, Koch P, Hänel M, Pfreundschuh M, Wilhelm M, Trümper L, Aulitzky WE, Bentz M, Rummel M, Sezer O, Müller-Hermelink HK, Hasenclever D, Löffler M (2009) Escalated-dose BEACOPP in the treatment of patients with advanced-stage Hodgkin's lymphoma: 10 Years of follow-up of the GHSG HD9 study. J Clin Oncol 27:4548-4554. https://doi.org/10.1200/JCO.2008.19.8820

2. Johnson P, Federico M, Kirkwood A, Fosså A, Berkahn L, Carella A, d'Amore F, Enblad G, Franceschetto A, Fulham M, Luminari S, O'Doherty M, Patrick P, Roberts T, Sidra G, Stevens L, Smith P, Trotman J, Viney Z, Radford J, Barrington S (2016) Adapted treatment guided by interim PET-CT scan in advanced Hodgkin's lymphoma. N Engl J Med 374:2419-2429. https://doi.org/10.1056/ NEJMoa1510093

3. Office for National Statistics (2019) 2011 Census: aggregate data. [data collection]. UK Data Service. SN: 7427,

4. Follows GA, Ardeshna KM, Barrington SF, Culligan DJ, Hoskin PJ, Linch D, Sadullah S, Williams MV, Wimperis JZ, The British Committee for Standards in Haematology (2014) Guidelines for the first line management of classical Hodgkin lymphoma. Br J Haematol 166:34-49. https://doi.org/10.1111/bjh.12878

5. Eichenauer DA, Engert A, André M et al (2014) Hodgkin's lymphoma: ESMO clinical practice guidelines for diagnosis, treatment and follow-up. Ann Oncol 25:iii70-iii75. https://doi.org/10.1093/ annonc/mdu181

6. Lad DP (2020) Making sense of cross-trial comparisons for the frontline treatment of advanced-stage Hodgkin lymphoma. Eur J Cancer 136:113-115. https://doi.org/10.1016/j.ejca.2020.05.012

7. Liu L, Giusti F, Schaapveld M, Aleman B, Lugtenburg P, Meijnders P, Hutchings M, Lemmens V, Bogaerts J, Visser O (2017) Survival differences between patients with Hodgkin lymphoma treated inside and outside clinical trials. A study based on the EORTC-Netherlands Cancer Registry linked data with 20 years of follow-up. Br J Haematol 176:65-75. https://doi.org/10.1111/ bjh.14379

8. Carde P, Karrasch M, Fortpied C, Brice P, Khaled H, Casasnovas O, Caillot D, Gaillard I, Bologna S, Ferme C, Lugtenburg PJ, Morschhauser F, Aurer I, Coiffier B, Meyer R, Seftel M, Wolf M, Glimelius B, Sureda A, Mounier N (2016) Eight cycles of ABVD versus four cycles of BEACOPPescalated plus four cycles of BEACOPPbaseline in stage III to IV, International Prognostic Score $\geq 3$, high-risk Hodgkin lymphoma: first results of the phase III EORTC 20012 intergroup trial. J Clin Oncol 34:2028-2036. https://doi.org/10.1200/JCO.2015.64.5648

9. Viviani S, Zinzani PL, Rambaldi A, Brusamolino E, Levis A, Bonfante V, Vitolo U, Pulsoni A, Liberati AM, Specchia G, Valagussa P, Rossi A, Zaja F, Pogliani EM, Pregno P, Gotti M, Gallamini A, Scalabrini DR, Bonadonna G, Gianni AM (2011) ABVD versus BEACOPP for Hodgkin's lymphoma when highdose salvage is planned. N Engl J Med 365:203-212. https://doi. org/10.1056/NEJMoa1100340

10. Mounier N, Brice P, Bologna S, Briere J, Gaillard I, Heczko M, Gabarre J, Casasnovas O, Jaubert J, Colin P, Delmer A, Devidas A, Bachy E, Nicolas-Virelizier E, Aoudjhane A, Humbrecht C, Andre M, Carde P, Lymphoma Study Association (LYSA) (2014) ABVD ( 8 cycles) versus BEACOPP ( 4 escalated cycles $\geq 4$ baseline): Final results in stage III-IV low-risk Hodgkin lymphoma (IPS 0-2) of the LYSA H34 randomized trial. Ann Oncol 25:1622-1628. https:// doi.org/10.1093/annonc/mdu189

11. Skoetz N, Trelle S, Rancea M, Haverkamp H, Diehl V, Engert A, Borchmann P (2013) Effect of initial treatment strategy on survival of patients with advanced-stage Hodgkin's lymphoma: a systematic 
review and network meta-analysis. Lancet Oncol 14:943-952. https://doi.org/10.1016/S1470-2045(13)70341-3

12. Borchmann P, Goergen H, Kobe C et al (2017) PET-guided treatment in patients with advanced-stage Hodgkin's lymphoma (HD18): final results of an open-label, international, randomised phase 3 trial by the German Hodgkin Study Group. Lancet:1-13. https://doi.org/10.1016/S0140-6736(17)32134-7

13. Casasnovas RO, Bouabdallah R, Brice P, Lazarovici J, Ghesquieres H, Stamatoullas A, Dupuis J, Gac AC, Gastinne T, Joly B, Bouabdallah K, Nicolas-Virelizier E, Feugier P, Morschhauser F, Delarue R, Farhat H, Quittet P, Berriolo-Riedinger A, Tempescul A, Edeline V, Maisonneuve H, Fornecker LM, Lamy T, Delmer A, Dartigues P, Martin L, André M, Mounier N, Traverse-Glehen A, Meignan M (2019) PET-adapted treatment for newly diagnosed advanced Hodgkin lymphoma (AHL2011): a randomised, multicentre, non-inferiority, phase 3 study. Lancet Oncol 20:202215. https://doi.org/10.1016/S1470-2045(18)30784-8

14. Kreissl S, Goergen H, Kobe $\mathrm{C}$ et al (2020) PET-guided treatment in patients with advanced-stage Hodgkin lymphoma: follow-up analysis of PET-2-negative patients in the HD18 Trial by the German Hodgkin Study Group. HemaSphere 4:69
15. Anderson RA, Remedios R, Kirkwood AA, Patrick P, Stevens L, Clifton-Hadley L, Roberts T, Hatton C, Kalakonda N, Milligan DW, McKay P, Rowntree C, Scott FM, Johnson PWM (2018) Determinants of ovarian function after response-adapted therapy in patients with advanced Hodgkin's lymphoma (RATHL): a secondary analysis of a randomised phase 3 trial. Lancet Oncol 19: 1328-1337. https://doi.org/10.1016/S1470-2045(18)30500-X

16. Martin L, Dartigues P, Bouabdallah R et al (2019) PET-adapted treatment for newly diagnosed advanced Hodgkin lymphoma (AHL2011): a randomised, multicentre, non-inferiority, phase 3 study. Lancet Oncol 20:202-215. https://doi.org/10.1016/s14702045(18)30784-8

17. Santarsieri A, Sturgess K, Brice P et al (2020) Escalated BEACOPDac as first line therapy in 100 Hodgkin lymphoma patients reduces red cell transfusion requirements and may shorten time to menstrual period recovery whilst maintaining efficacy compared to escalated BEACOPP. Br J Haematol 189:97-294. https:// doi.org/10.1111/bjh.16638

Publisher's note Springer Nature remains neutral with regard to jurisdictional claims in published maps and institutional affiliations.

\section{Affiliations}

\section{James Russell ${ }^{1}$ (D) Angela Collins ${ }^{2} \cdot$ Alexis Fowler $^{3} \cdot$ Mamatha Karanth $^{4} \cdot$ Chandan Saha $^{1} \cdot$ Suzanne Docherty $^{2}$. Joseph Padayatty ${ }^{1}$. Kyaw Maw ${ }^{5}$. Isabel Lentell ${ }^{4}$. Lisa Cooke ${ }^{6}$. Andrew Hodson ${ }^{7}$. Nimish Shah ${ }^{2}$. Shalal Sadullah ${ }^{5}$. Nicholas Grigoropoulos ${ }^{1} \cdot$ Wendi Qian ${ }^{1} \cdot$ Amy A. Kirkwood ${ }^{8} \cdot$ Benjamin J. Uttenthal $^{1} \cdot$ Peter Johnson ${ }^{9}$. George A. Follows ${ }^{1}$}

1 Department of Haematology, Addenbrooke's Hospital, Cambridge University Hospitals NHS Foundation Trust, Hills Road, Cambridge CB2 0QQ, UK

2 Department of Haematology, Norfolk and Norwich University Hospital, Norfolk and Norwich University Hospitals NHS Foundation Trust, Colney Lane, Norwich NR4 7UY, UK

3 Department of Haematology, Peterborough City Hospital, Peterborough and Stamford Hospitals NHS Foundation Trust, Edith Cavell Campus, Bretton Gate, Peterborough PE3 9G, UK

4 Department of Haematology, West Suffolk Hospital, West Suffolk NHS Trust, Hardwick Lane, Bury St Edmunds IP33 2QZ, UK
5 Department of Haematology, James Paget University Hospital, James Paget University Hospitals NHS Foundation Trust, Lowestoft Road, Gorleston-on-Sea, Great Yarmouth NR31 6LA, UK

6 Department of Haematology, The Queen Elizabeth Hospital, King's Lynn NHS Foundation Trust, Gayton Rd, King's Lynn PE30 4ET, UK

7 Department of Haematology, Ipswich Hospital, Ipswich Hospital NHS Trust, Heath Rd, Ipswich IP4 5PD, UK

8 Cancer Research UK and University College London Cancer Trials Centre, University College London, 90 Tottenham Court Road, London W1T 4TJ, UK

9 Cancer Research UK Centre, University of Southampton, University Road, Southampton SO17 1BJ, UK 